\title{
FZR-197
}

October 1997

Preprint

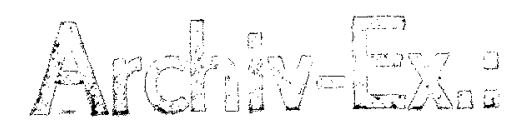

D.V. Kamanin, W. Wagner and H.-G. Ortlepp

\section{A method for the intrinsic calibration of CsI(TI) detectors}


Herausgeber:

FORSCHUNGSZENTRUM ROSSENDORF

Postfach 510119

D-01314 Dresden

Telefon (03 51) 2600

Telefax (03 51) 2690461

Als Manuskript gedruckt

Alle Rechte beim Herausgeber 


\title{
A method for the intrinsic calibration of $\mathrm{CsI}(\mathrm{Tl})$ detectors
}

\author{
D.V. Kamanin ${ }^{\dagger}$, W. Wagner, and H.-G. Ortlepp \\ ${ }^{\dagger}$ Absent on leave from Joint Institute for Nuclear Research, \\ 141980 Dubna, Moscow Reg., Russia \\ Submitted to \\ "Nuclear Instruments and Methods in Physical Research" \\ Section $A$
}

\begin{abstract}
A method for the intrinsic energy calibration of photomultiplier-coupled CsI(Tl) detectors is described. A simple empirical model of the scintillation light pulse-shape of CsI(Tl) crystals for light charged particles has been applied to simulate the particle identification matrix as it follows from the pulse-shape analysis method. The calibration procedure for the large-area CsI(TI) detectors of the scintillator shell of the $4 \pi$-array FOBOS for ions with $Z<4$ at energies below $100 \mathrm{AMeV}$ is based on the energies of the particle punch-through points.
\end{abstract}

Keywords: scintillator, energy calibration, pulse-shape analysis.

Corresponding author:

D.V.Kamanin, Forschungszentrum Rossendorf e.V., Postfach 510119, D-01314, Dresden, Deutschland email: D.Kamanin@fz-rossendorf.de phone: +493512603376

Permanent address:

D.V.Kamanin, FLNR, Joint Institute for Nuclear Research, 141980 Dubna, Moscow region, Russia email: kamanin@nrsun.jinr.dubna.su fax: +70962165083 phone: +70962164625 


\section{Introduction}

The scintillation process has been studied, and photomultiplier-coupled scintillation detectors are widely used for the registration of ionizing radiation for about fifty years [1]. In particular, inorganic CsI(Tl) crystals have some anticipated features. They are mechanically rugged, plastic, easily machinable and only slightly hygroscopic. The relative compactness of $\mathrm{CsI}(\mathrm{Tl})$ counters due to the large stopping power of the scintillator, the high scintillation efficiency due to low quenching, and, last but not least, the medium market price make $\mathrm{CsI}(\mathrm{Tl})$ one of the most appropriate scintillating materials for the detection of light charged particles and medium mass ions in nuclear research [2,3]. Mainly these reasons defined the choice of $\mathrm{CsI}(\mathrm{Tl})$ for the scintillator shell [4] of the $4 \pi$-array FOBOS [5].

The light output of $\mathrm{CsI}(\mathrm{Tl})$ evinces a strong dependence not only on the energy $(E)$ of the incoming particles, but also on their atomic $(Z)$ and mass number $(A)$. Furthermore, the scintillation light pulse-shape is a complicate function of the stopping power $d E / d x$ (cf. ref. [6] and refs. therein).

Hence, much attention has already been paid to the problem of detector calibration. There are some common features in the scintillation light processing in spite of the different operation modes of the CsI(Tl) crystals (e.g. utilizing them in the usual regime or in a phoswich combination with other scintillators [7], in $\Delta E(\mathrm{Si})-E(\mathrm{CsI})$ telescopes [6, 8], for the $T O F-E$ (CsI) analysis [9, 10], applying photodiode read-out [11, 12] etc.). In particular, the pulse-shape analysis (PSA) method $[6,13]$ is used to search for the best particle separation and energy resolution for light charged particles (LCP) in a wide dynamical range.

The energy calibration of the responce is commonly based on the measurement of the total light output, but the dependence on the shaping time has also been considered [14]. Often a few calibration points are obtained by a direct exposition of the detector to radioactive sources or ion beams, exploiting in this case addidional $\triangle E(\mathrm{Si})$ or TOF information for further analysis. The energy calibration is then obtained by fitting the data with sophisticated empirical functions for the light output $L(E)[15,16]$ or $E(L)[7]$. Such functions can also be the result of analytical calculations based on models for the energy deposition along the ion track in the scintillator and the luminescence process [17].

The aim of the present work is to perform an intrinsic calibration of the CsI(Tl) detectors, used in the scintillator shell of FOBOS [4], for the LCP spectra in the absence of especially measured reference points. The only available information is given by the punch-through points (PTPs) of different LCPs, and the corresponding energy values are the maximal energies which can be deposited in the crystal by these particles. Moreover, the variant of PSA used at FOBOS for LCP separation does not deliver the total light output, i.e. the total integral of the light pulse, but only two partial ones. We, therefore, applied a rather simple model for the pulse-shape of the CsI(TI) light pulse in dependence on $(Z, A)$ of the incoming ion and its energy $(E)$ and, further, on simulated the particle identification matrix (PIM) as it follows from the application of the PSA method under the real experimental conditions. Scaling properties have been found which are very useful for the calibration procedure of the altogether $210 \mathrm{CsI}(\mathrm{TI})$ detectors of FOBOS. In the following, the calibration method is described in detail. A first attempt to apply such a method has been published earlier in ref. [18].

\section{Experimental set-up}

The $4 \pi$-spectrometer FOBOS [5] is a logarithmic detector device for the study of heavy-ion induced reactions in the energy range of $10 \div 100 \mathrm{AMeV}$. It consists of three consecutive shells 
of particle detectors and a more granular forward array [19]. The inner two detector shells consist of 30 position-sensitive avalanche counters (PSACs) and 30 axial Bragg ionization chambers (BICs). Mosaic arrangements, each consisting of 7 hexagonal-shaped $\mathrm{CsI}(\mathrm{Tl})$ crystals, are placed behind the BICs constituting the outer detector shell. One PSAC, one $\mathrm{BIC}$ and $7 \mathrm{CsI}(\mathrm{Tl})$ counters form a detector module [20].

A single CsI(TI) detector unit [21] consists of a large-area $\left(260 \mathrm{~cm}^{2}\right.$ or $\left.146 \mathrm{~cm}^{2}\right)$ crystal (MONOCRYSTALREACTIV Company, Kharkov, Ukraine) and a hollow light guide coupled to a spectroscopic photomultiplier (SPM) (type FEU-173, $\oslash=170 \mathrm{~mm}$ or FEU-167, $\oslash=120 \mathrm{~mm}$; EKRAN Company, Novosibirsk, Russia). The front side of the crystal is polished and, in order to enhance the light output, covered with a $1.5 \div 3 \mu \mathrm{m}$ thick reflector foil of aluminized Mylar mounted at a distance of $3 \mathrm{~mm}$ from the surface. The rear side of the crystal is rough. The content of the Tl activator amounts to $0.07 \div 0.08 \%$ being found as an optimum, considering the scintillation efficiency and PSA properties for LCPs. The hollow light guide has a diffuse-reflecting (90\% reflectivity) inner surface, and diminishes the position dependence of the light collection to about $5 \%$. The energy resolution for $5.5 \mathrm{MeV}$ $\alpha$-particles is typically $6 \div 7 \%$ for collimated particles and $9 \%$, if the entire surface of the crystal is illuminated [21].

The thickness of the CsI(Tl) crystals in forward-positioned detectors at polar angles of $\vartheta=23^{\circ} \div 52^{\circ}$ amounts to $15 \mathrm{~mm}$, the other part of the scintillator shell in the angular range of $\vartheta=53^{\circ} \div 157^{\circ}$ consists of $10 \mathrm{~mm}$ thick crystals. The total covered solid angle is $5.6 \mathrm{sr}$, but the effective solid angle amounts to about 4 sr because of the limited transparency of the inner detector shells [5].

The current signals from the SPMs are split and integrated by 96-channel QDCs (C.A.E.N. CIAFB F683C) within two time gates in accordance with the pulse processing necessary for the PSA [22]. Due to the particle- and energy-dependent decay constants of CsI(Tl) $([6,13]$ and refs. therein), the best LCP separation in the PIM is observed with the time gates $\Delta t_{\text {fast }}=0 \div 400 \mathrm{~ns}$ and $\Delta t_{\text {slow }}=1600 \div 4600 \mathrm{~ns}$. Initial values for these gates were estimated by simulations like those made in ref. [6]. The real experimental conditions (timing, trigger logics etc.) in measurements with the whole detector array may require some modification of these time gates.

\section{Calibration method}

Under the assumption that all CsI(Tl) crystals of the scintillator shell have similar properties (that was guaranteed by the manufacturer), all SPMs are operated in a linear regime, and all signals are processed in a unique manner, one expects that all PIMs look similar, and one can sum them up into one PIM after some linear transformation accounting for different gain constants. Therefore, we scaled the individual PIMs to each other. The summed PIM is shown in fig. 1. Indeed, resolved particle branches occur for the $H$ and $H e$ isotopes, also for ${ }^{6} \mathrm{He}$ and ${ }^{8} \mathrm{He}$. Furthermore, particle branches of heavier particles are clearly seen. Usually, these branches are very weak in the PIMs of the individual detectors due to low statistics. Note that the scaling procedure does not lead to loss of particle resolution.

The maximal energies which can be deposited in the given CsI(TI) crystals (PTPs) by $H, H e$ and $L i$ isotopes were calculated using the stopping power code STOPPOW [23], and are given in Table 1. Particles with higher energies are not stopped in the crystals. They penetrate them, and the deposited energy becomes successively lower with increasing incident energy. Hence, the particle branches, after reaching the PTPs, turn backward (cf. fig. 1. ) approaching the branch of low-ionizing particles (electrons, $\gamma$-rays). The PTPs are well pronounced in the PIMs of forward-positioned detectors. 


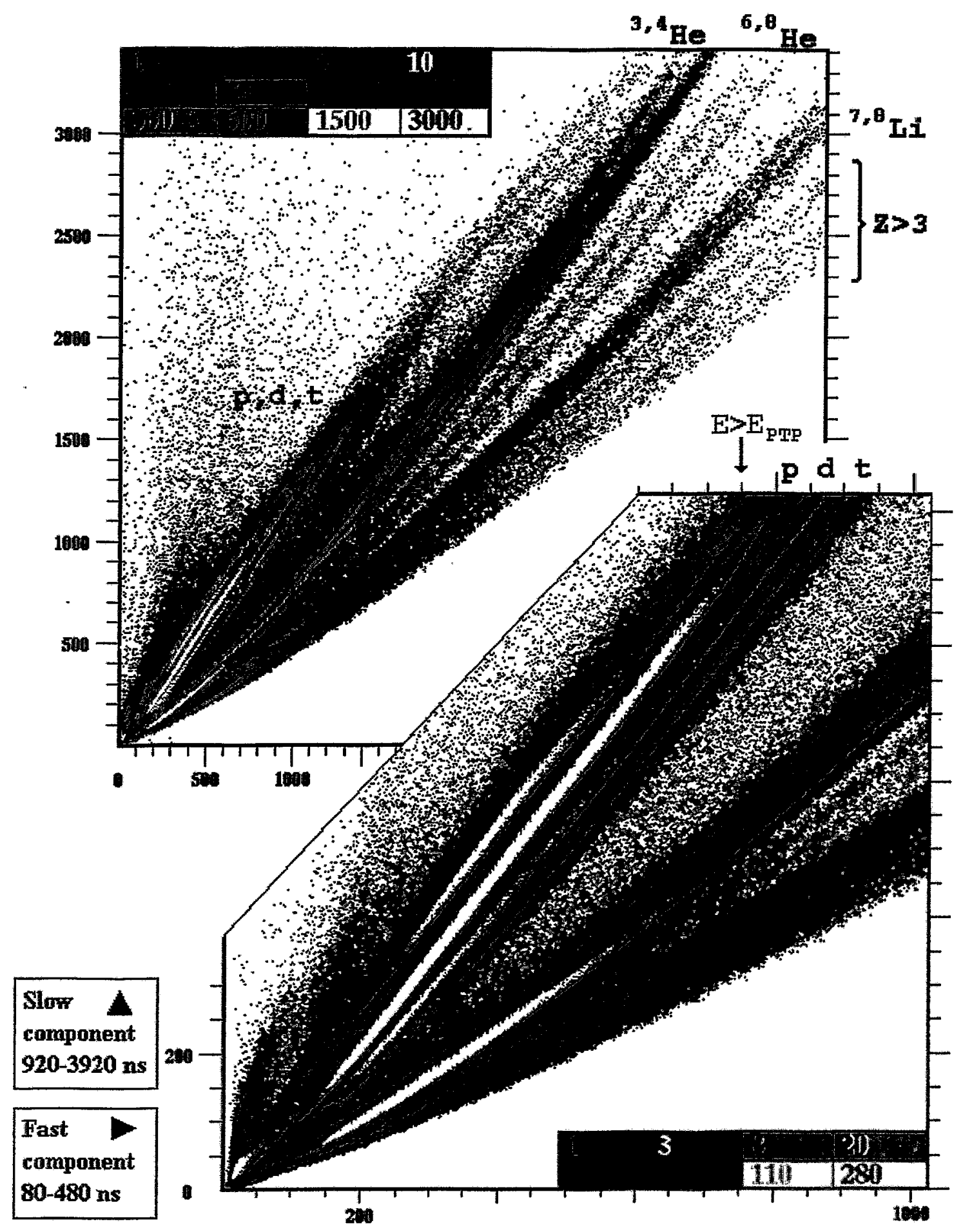

Figure 1: Summed particle identification matrix (PIM) for the $\mathrm{CsI}(\mathrm{TI})$ detectors positioned at $\vartheta_{\text {lab }}=$ $28^{\circ} \div 47^{\circ}(\Delta \Omega=0.4 \mathrm{sr})$. This PIM represents $40 \%$ of the entire data body recorded for the reaction ${ }^{14} \mathrm{~N}(53 \mathrm{AMeV})+{ }^{232} \mathrm{Th}$. The energy scale extends to $\approx 300 \mathrm{MeV}$. 
The CsI(TI) crystals are manufactured with precision of $0.1 \mathrm{~mm}$. Therefore, the PTPS can be treated as intrinsic energy reference points suitable for calibration purposes.

Table 1: Maximum energy losses of light ions in the CsI(Tl) crystals of the scintillator shell of FOBOS (PTPs)

\begin{tabular}{|c|cc|cc|}
\hline \multirow{2}{*}{ Particle } & \multicolumn{2}{|c|}{$A \mathrm{MeV}$} & \multicolumn{2}{c|}{$\mathrm{MeV}$} \\
\cline { 2 - 5 } & $10 \mathrm{~mm}$ & $15 \mathrm{~mm}$ & $10 \mathrm{~mm}$ & $15 \mathrm{~mm}$ \\
\hline$p$ & 51.0 & 64.5 & 51.0 & 64.5 \\
$d$ & 34.2 & 43.2 & 68.3 & 86.4 \\
$t$ & 27.0 & 34.2 & 80.9 & 102.5 \\
\hline${ }^{3} \mathrm{He}$ & 60.3 & 76.1 & 180.8 & 228.4 \\
${ }^{4} \mathrm{He}$ & 51.1 & 64.5 & 204.2 & 258.0 \\
${ }^{6} \mathrm{He}$ & 40.4 & 51.1 & 242.3 & 306.3 \\
${ }^{8} \mathrm{He}$ & 34.2 & 43.2 & 273.4 & 345.8 \\
\hline${ }^{6} \mathrm{Li}$ & 64.6 & 81.5 & 387.3 & 489.1 \\
${ }^{7} \mathrm{Li}$ & 59.1 & 74.6 & 413.4 & 522.1 \\
\hline
\end{tabular}

The clear indication that PTPs are really reliable is given in fig. 2. The relative error of the identification of the coordinates of the PTPs in the PIMs of individual detectors is typically about $2 \%$. The scaling procedure, therefore, is based on PIMs with well pronounced PTPs. It brings the particle branches, and naturally also the PTPs, of all CsI(TI) detectors to superposition.

Especially in the detectors positioned in the backward hemisphere of FOBOS, the PTPS are weakly pronounced or absent at all. To add these PIMs to the summed PIM too, we applied the following method. We first constructed an "ideal" PIM out of such individual PIMs, where a good particle resolution is observed and the PTPs (mainly those of the $H$ isotopes) are clearly pronounced as well. A special procedure was developed utilizing also the shapes of the particle branches in the PIMs for scaling purpose. It is based on the simulation of the PIM as it followes from the application of the PSA method. Simultaneously, relative energy scales for the individual particle branches are generated. The normalization of the simulated PIM at the PTPs then delivers the absolute energy scales. By a suitable variation of the energy- and particle-dependent parameters being ingredients of the model of the scintillation light pulse-shape, the experimentally observed shapes of the particle branches in the PIM can easily be generated. The obtained relative energy scales can then be used for the scaling of the PIMs without PTPs for adding them to the "ideal" PIM. In the following, this method is described in detail.

\section{Simulation of the particle identification matrix}

The simulation of the PIM as it follows from the PSA method used is based on the approximation of the CsI(Tl) scintillation light pulse-shape $\mathrm{L}(\mathrm{t})$ (eq. (1)) by three exponential functions with the characteristic time constants $\tau_{i}$; a few hundreds of nanoseconds for the "fast" light component $\left(\tau_{\text {fast }}\right)$, a few thousands of nanoseconds for the "slow" light component $\left(\tau_{\text {slow }}\right)$, and $10 \div 100$ ns to take into account the pulse rise-time $\left(\tau_{\text {front }}\right)$.

$$
L(t)=\frac{h_{\text {slow }}}{\tau_{\text {slow }}} \exp \left(-\frac{t}{\tau_{\text {slow }}}\right)+\frac{h_{\text {fast }}}{\tau_{\text {fast }}} \exp \left(-\frac{t}{\tau_{\text {fast }}}\right)-\frac{h_{\text {front }}}{\tau_{\text {front }}} \exp \left(-\frac{t}{\tau_{\text {front }}}\right)
$$




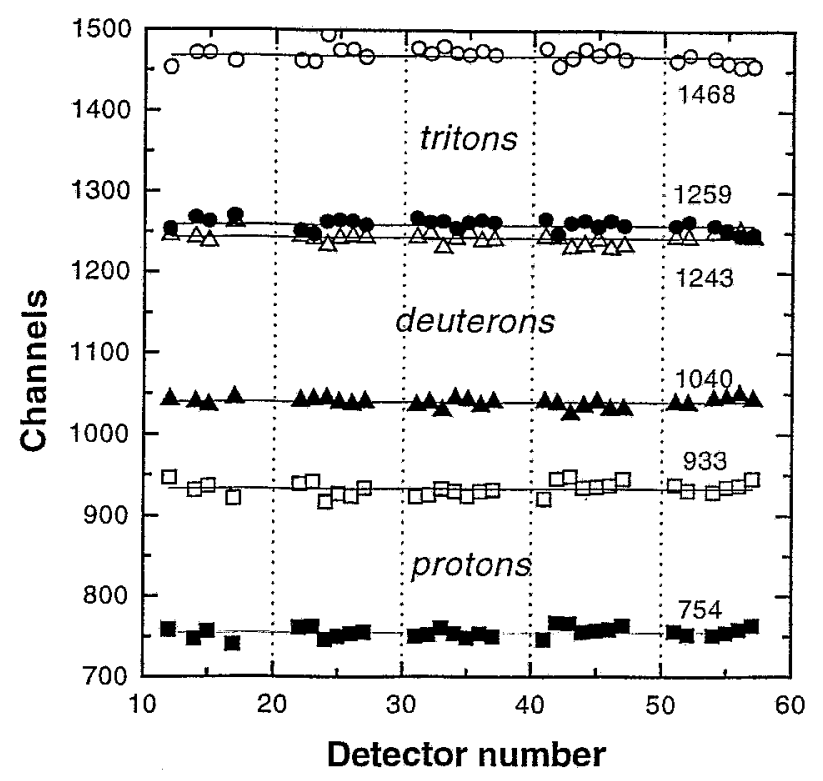

Figure 2: Coordinates of punch-through points in individual CsI(TI) detectors after application of the scaling procedure. Solid symbols denote the coordinates $L_{f a s t}$, open symbols denote the coordinates $L_{\text {slow }}$.

$h_{\text {fast }}$ and $h_{\text {slow }}$ denote the magnitudes of the two light components. Coordinates of the PIM are the integrals $L_{\text {fast }}$ and $L_{\text {slow }}$ of $L(t)$ taken for the hardware-set time gates $\Delta t_{i}$. We used values of $\Delta t_{\text {fast }}=80 \div 480 \mathrm{~ns}$ and $\Delta t_{\text {slow }}=920 \div 3920 \mathrm{~ns}$ (fig. 3 ). The time constant $\tau_{\text {slow }}$ is known to be nearly particle-independent, and takes values in the range of $4 \div 7 \mu \mathrm{s}$. The ratio $R=h_{\text {slow }} / h_{\text {fast }}$ as well as the decay time constant of the fast component $\tau_{\text {fast }}$ are decreasing functions of the stopping power $d E / d x$ ([6] and refs. therein). The function $\tau_{\text {fast }}$ shows some saturation effect near a stopping power of $1000 \mathrm{MeV} / \mathrm{cm}$ [24].

The properties of $\mathrm{CsI}(\mathrm{Tl})$ crystals strongly depend on their quality and $\mathrm{Tl}$ concentration. To get analytic expressions for the dependence of $R$ and $\tau_{\text {fast }}$ on $E$ and the type of particle $(Z, A)$, we fitted appropriate empirical functions $R(E, Z, A)$ and $\tau_{f a s t}(E, Z, A)$ to the experimental data given in ref. [6]. The dependence on the type of particle is given in terms of the quenching parameter $q=A Z^{2}$. To limit the number of fitting parameters, the following expressions seemed to us to be suitable ones:

$$
\begin{gathered}
R(E, q)=\frac{R_{0}}{q^{1 / 4}}\left[1-\exp \left(-\frac{d}{q^{1 / 4}} E^{Q}\right)\right] \\
\tau_{\text {fast }}(E, q)=\tau_{0}+\frac{\tau_{I}}{q^{0.1834}}\left[1-\exp \left(-\frac{d}{q^{1 / 4}} E^{Q}\right)\right]
\end{gathered}
$$

where the fitting parameters took values of $\tau_{0}=365 \mathrm{~ns}$. $\tau_{1}=3323 \mathrm{~ns}, R_{0}=4$, and $d=0.081$. The parameter $Q$ was found to be slightly dependent on $q$. For extrapolation purposes, we expressed it also as a function of $q$

$$
Q(q)=0.285\left[1-\exp \left(-\frac{q}{0.611}\right)\right] q^{0.1022}
$$




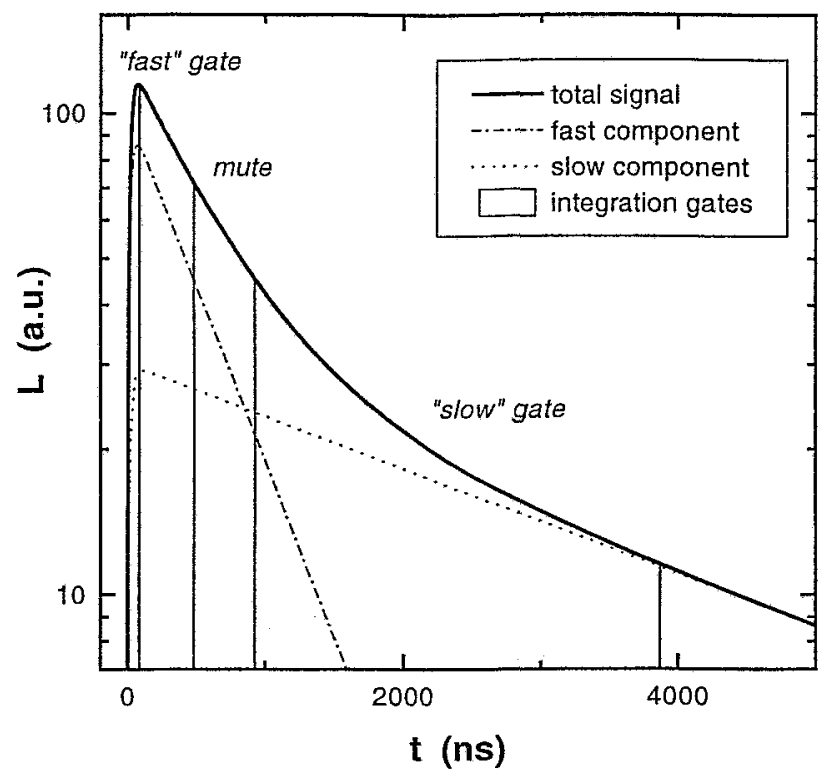

Figure 3: Calculated shape of a scintillation light pulse of CsI(Tl), constructed by superposition of the fast and slow components of an $\alpha$-particle of $50 \mathrm{MeV}$ energy. The time gates are shown as they were set in the experiment.

The functions $R(E, q)$ and $\tau_{f a s t}(E, q)$ must not necessarily represent the best fit for each particle type individually, but they describe the general trend sufficiently well (fig. 4 ).

If the ratio of the light components $(R)$ is given, the absolute values for $h_{\text {fast }}$ and $h_{\text {slow }}$ can be derived from the normalization of the total integral of $L(t)$ to the total light output $L(E)$

$$
\int_{0}^{\infty} L(t, E, Z, A) d t=h_{\text {slow }}+h_{\text {fast }}-h_{\text {front }} .
$$

The latter is set to be equal to the expression given in ref. [15],

$$
L(E)=S\left[E-a(Z, A) \ln \left(\frac{E}{a(Z, A)}+1\right)\right]
$$

where $E$ is the energy deposited in the $\mathrm{CsI}(\mathrm{Tl})$ crystal, $a(Z, A)$ is the quenching constant, and $S$ is the scintillation efficiency. By the condition $L(t=0)=0$ the value of $h_{\text {front }}$ is connected with $R$ and the time constants and it can be easily estimated.

Performing the simulation of the PIM for given types of particles, intervals of incident energy and time gates $\left(\Delta t_{\text {fast }} \Delta t_{\text {slow }}\right)$, we simultaneously get the relative calibration curves $L_{\text {fast }}(E)$ and $L_{\text {slow }}(E)$ for every particle branch. The simulated PIM is shown in fig. 5 . The normalization of the simulated PIM to the "ideal" PIM (fig. 1) with reference to the positions of the PTPs delivers the particle-dependent absolute energy scales. The shapes of the particle branches in the simulated PIM can principally be modified to approach the experimentally observed ones (fig. 1) by slight variations of the parameters of eqs. (2) and (3), accouting in this manner for the properties of the CsI(TI) crystals used. 

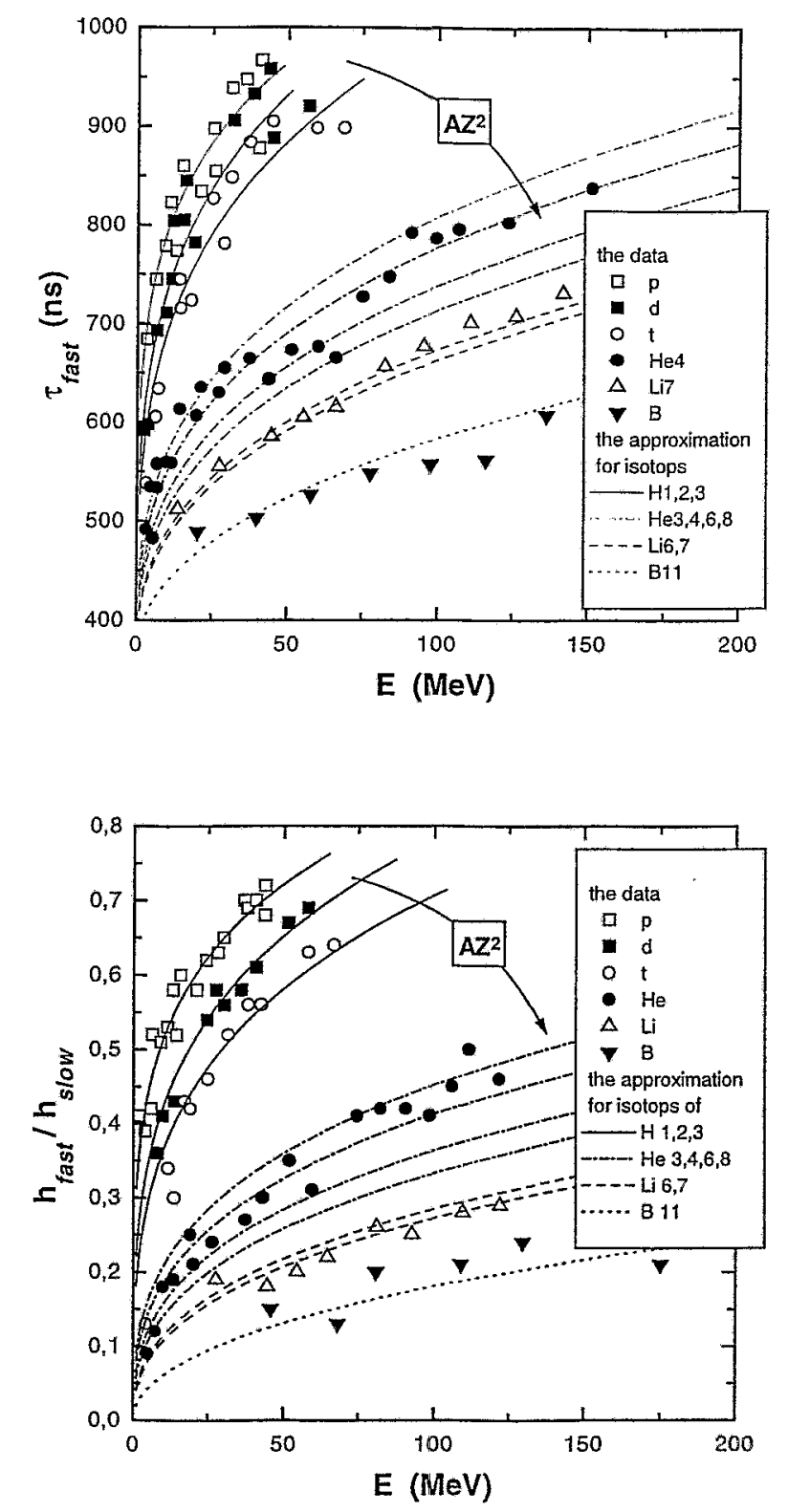

Figure 4: Comparison of $\mathrm{CsI}(\mathrm{Tl})$ data of ref. [6] (symbols) with our approximations (lines) basing on a quenching parameter systematics. The arrows show the rise of the decay time of the fast light component (upper panel) and of the ratio between the magnitudes of the slow and fast light components (lower panel) with $A Z^{2}$ as a function of the energy of an incident particle. 


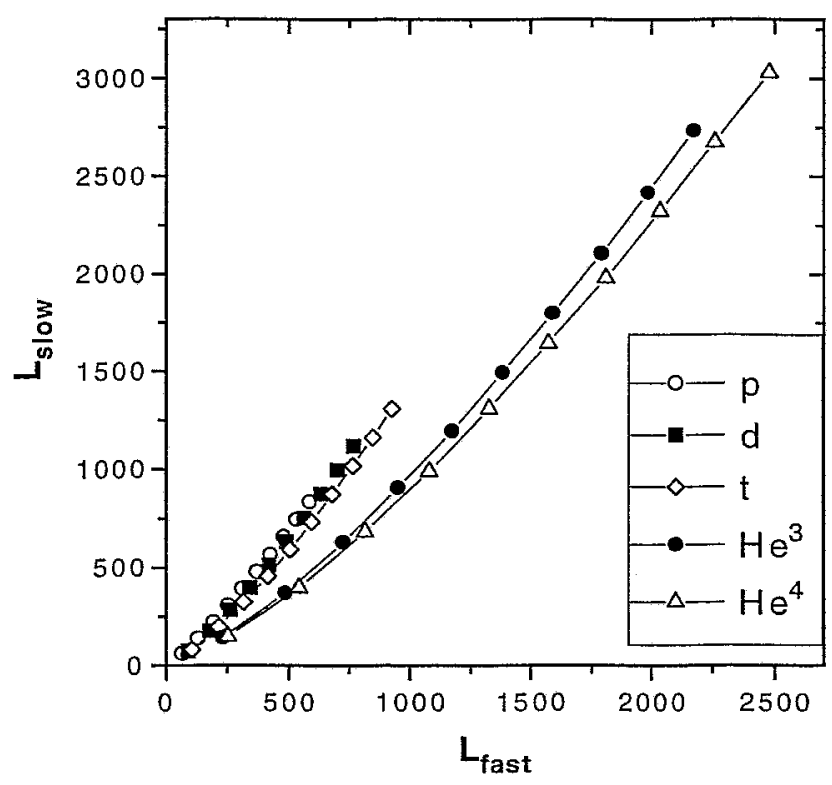

Figure 5: Particle identification matrix $L_{\text {slow }}$ vs $L_{\text {fast }}$ of a CsI(Tl) detector simulated for $\Delta t_{\text {fast }}=80 \div$ $480 n s, \Delta t_{\text {slow }}=920 \div 3920 \mathrm{~ns}$, and $\Delta \tau_{\text {slow }}=4 \mu \mathrm{s}$.

Since $\Delta t_{\text {fast }}<\Delta t_{\text {slow }}$, the contribution of noise in the measured values of $L_{\text {fast }}$ is significantly less than in $L_{\text {slow }}$. On the other hand, $L_{\text {slow }}$ is less sensitive to small energies. We used $L_{f a s t}(E)$ for the calibration of the spectra of LCPs within the entire dynamical range. The calibration curves for both light components with respect to the time gates used are shown in fig. 6.

The energy losses of the particles on the flight path from the target to the CsI(T1) crystals in the penetrated detector materials (i.e. the PSAC foils, the windows and the gas volume of the BIC, the Mylar reflector foil) were calculated using the code STOPPOW [23]. The dead layer at the surface of the CsI(TI) crystal [25] is very thin and was neglected. A useful empirical expression for the correction of the energy losses has been deduced (fig. 7),

$$
E_{t}=\left[\left(E_{d}+E_{0}\right)^{\alpha}+C^{\alpha}\right]^{1 / \alpha}-E_{0}
$$

where $E_{t}$ and $E_{d}$ are the energies of the particles at the target and in the CsI(Tl) crystal, respectively, and $E_{0}, C$ and $\alpha$ are fitting parameters. The function $E_{t}\left(L_{f a s t}\right)$ can be written in the form

$$
f(x)=a x^{b}+\frac{c_{1} x}{d_{1}+x}+\frac{c_{2} x}{d_{2}+x} .
$$

A special test measurement was carried out to check the reliability of the calibration procedure. In this measurement a BIC was used as $\Delta E$ detector, and the CsI(Tl) scintillator measured the residual energy [22]. In spite of the special operation mode of the BIC, this test was more qualitative than quantitative because of the large uncertainties due to the small $\Delta E$ signals of $\alpha$-particles, the limited dynamical range available, and the generally low registration efficiency of the BICs for LCPs.

An additional check was performed by comparing the results for the $10 \mathrm{~mm}$ thick crystals with those of the $15 \mathrm{~mm}$ thick ones. Such comparison is efficient, if the transparency of 


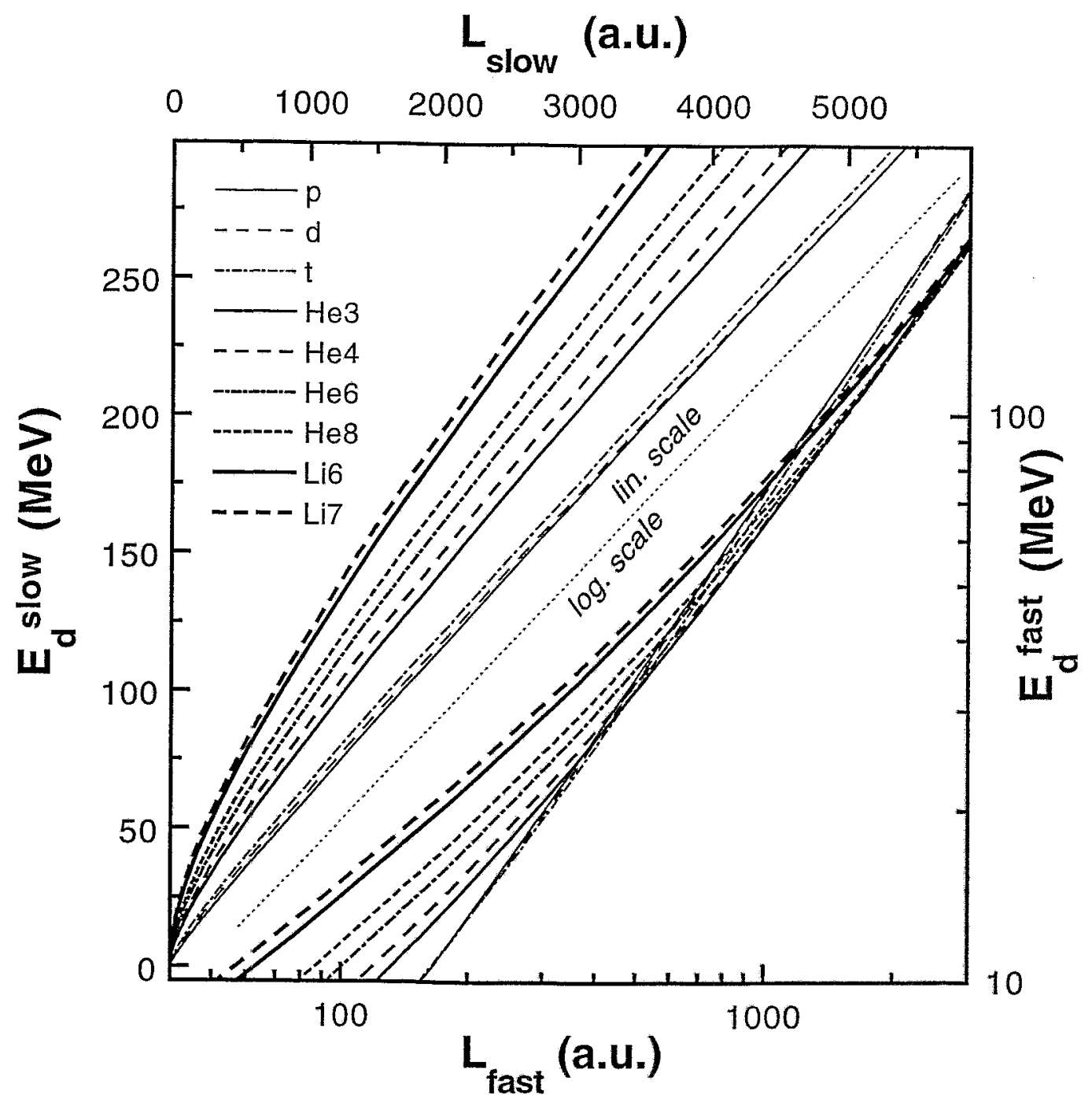

Figure 6: Simulated calibration curves for the slow (upper left corner) and fast (lower right corner) PSA components recorded for time gates $\Delta t_{\text {fast }}=80 \div 480$ ns and $\Delta t_{\text {slow }}=920 \div 3920$ ns, respectively. For better visuality, the fast component is plotted in a logarithmic scale. 


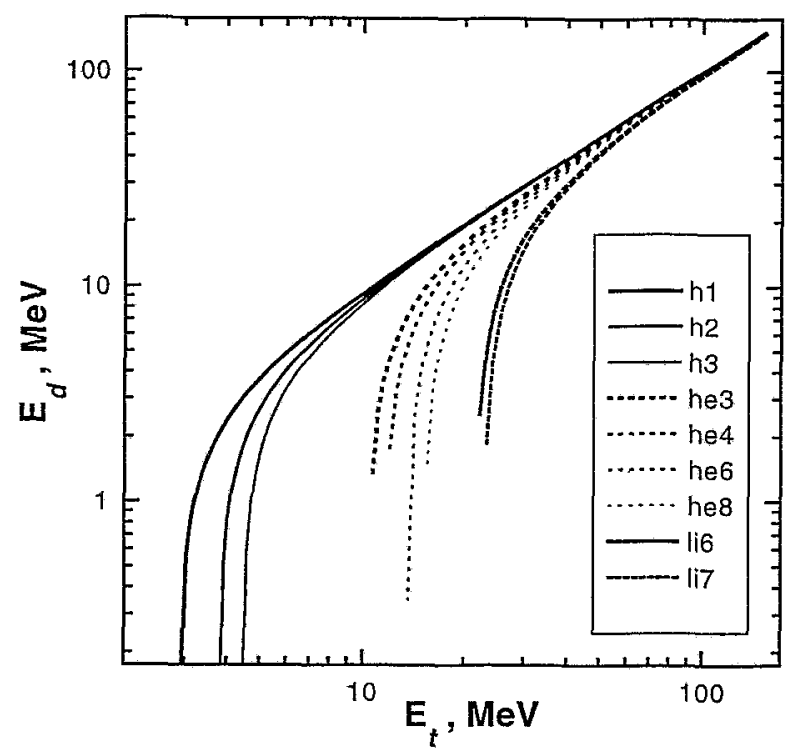

Figure 7: Energy losses of the LCPs on their flight path from the target to the CsI(TI) detector. They were calculated using the code STOPPOW [23].

the CsI(Tl) crystals is high enough. The PIM of the thick crystal was scaled to that of the thinner one by means of a scaling procedure which considered the shapes of the particle branches (see chap. 5). The result is shown in fig. 8.

\section{Shape-scaling method}

As already mentioned above, PTPs are not available in some cases. There are mainly two reasons for this. First, the energies of the particles emitted into the backward hemisphere (in the lab-system) are lower due to effects of transferred linear momentum in the nuclear reaction. Secondly, a possible mismatch of gain adjustment can occur due to low counting rates. If the PIM is not distorted by other influences, it can be scaled to the "ideal" one by use of the shapes of the particle branches. The idea is demonstrated in the following.

Two lines of different curvature, e.g.

$$
y=a_{i} x^{b_{i}}
$$

can unambiguously be scaled to pass through two arbitrarily chosen points in some area $(x, y)$ by means of the transformation

$$
k_{y} y=a_{i}\left(k_{x} x\right)^{b_{i}}
$$

where the coefficients $k_{x}$ and $k_{y}$ can be defined analytically.

Such a transformation cannot be applied directly to the experimental PIMs, because the particle branches are crooked insignificantly. Thus small discrepancies of the shapes of the particle branches in the PIMs together with the effect of noise lead to intolerable uncertainties. Non-linearities of such kind introduce variations of the coefficients $b_{i}$ of about $13 \%$ and $26 \%$ for protons and $\alpha$-particles, respectively. 


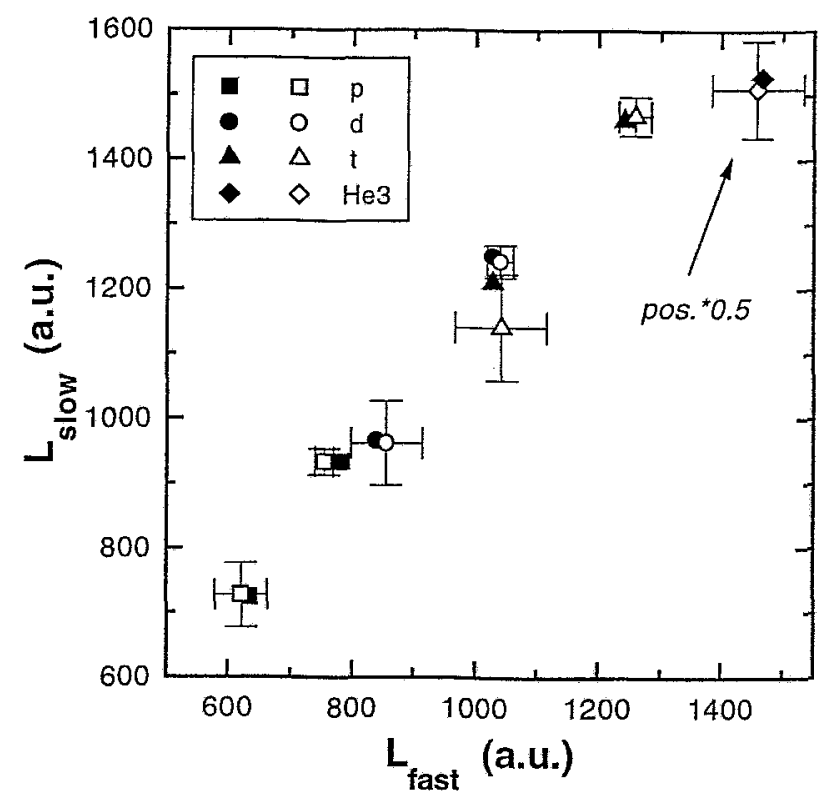

Figure 8: Comparison of the simulated coordinates of the punch-through points (solid symbols) with the measured ones (open symbols). The points with larger error bars (except for ${ }^{3} \mathrm{He}$ ) correspond to a $10 \mathrm{~mm}$ thick crystal. They were obtained applying the shape-scaling. The points for ${ }^{3} H e$ are, for convenience, multiplied by a factor of 0.5 .

Therefore, we constructed a model of the "ideal" PIM using empirical functions for the description of the particle branches,

$$
F_{\text {ideal }}\left(L_{\text {fast }_{i}}, L_{\text {slowi }}, A_{i}, Z_{i}\right)=0
$$

where the index $i$ denotes the type of particle $(Z, A)$. The coefficients $k_{\text {fust }}$ and $k_{s i o w}$ are found by minimization of a $\chi^{2}$-like functional

$$
\sum_{i} F_{\text {ideal }}\left(k_{\text {fast }} L_{\text {fast }}, k_{\text {slow }} L_{\text {slowi }}, A_{i}, Z_{i}\right)=\min
$$

Of course, a suitable choice of $F_{\text {ideal }}$ is important. Functions of the form like eq. (9) in general fit the particle branches well. But they fail in the region of low energy, that becomes critical for the PIMs of backward-positioned detectors. Therefore, we used functions of the form of eq. (8) for $L_{\text {slow }}$ and $L_{\text {fast }}$. Then the corresponding functional becomes

$$
\chi^{2}=\sum_{j}\left(k_{y} Y_{j}-F\left(k_{x} X_{j}\right)\right)^{2}
$$

where $X$ and $Y$ are the two components of the PSA, $j$ denotes a point in the experimental PIM, and $F$ represents any function $X=F(Y, Z, A)$ of the type like eq. (1I).

There is also another way to define the $\chi^{2}$-like functional.

$$
\chi^{2}=\sum_{j}\left(Y_{j}-F\left(k_{x} X_{j}\right) / t_{3 j}\right)^{2}
$$


The points $\left(X_{j}, Y_{j}\right)$ are arbitrarily but nearly eqidistantly scanned along the entire particle branches of the PIM. The coefficients $k_{x}$ and $k_{y}$ are then obtaind numerically by setting the derivatives $d \chi^{2} / d k_{x}$ and $d \chi^{2} / d k_{y}$ equal to zero.

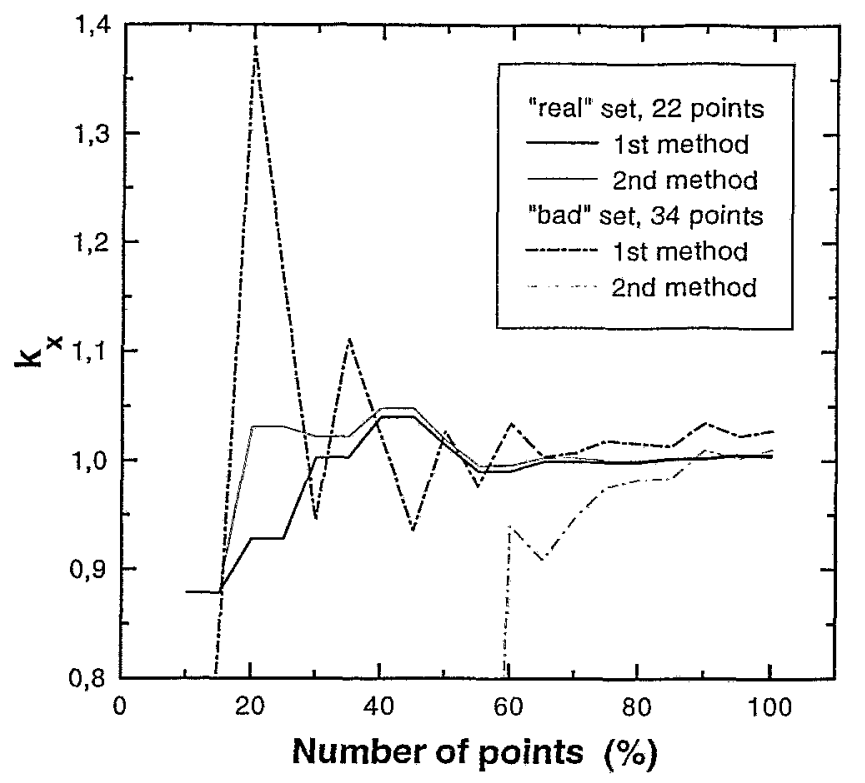

Figure 9: Coefficients obtained for the shape-scaling of the "ideal" particle identification matrix to itself. They are based on the particle branches of protons and $\alpha$-particles. The abscissa corresponds to that part of points which were used for scaling, counted from the side of lower energy. The "real" set was scanned as usual, and the "bad" set was scanned under the assumption of some oscillation of the points around the particle branch within $\pm 2 \%$ of the actual value of the coordinates. The 1st and 2nd method of $\chi^{2}$-calculation is in correspondence with their order of description in the text.

The solutions of eqs. (13) and (14) are found to be identical for PIMs with well resolved particle branches. They also perfectly describe the PIMs with pronounced PTPs. Discrepancies with respect to less resolved PIMs or PIMs without pronounced PTPs are significantly larger for eq. (14) than for eq. (13), but a comparison of the coefficients obtained for PIMs with and without PTPs cannot prefer one of these equations. This fact indicates some limits of such a procedure. Nevertheless, the precision of the shape-scaling method was estimated to be about $5 \%$ with respect to the energy scale. This is illustrated in fig. 9.

\section{Discussion}

Finally, we estimated the precision of the calibration procedure described in this work as being better than $10 \%$, i.e. of the order of the energy resolution of the $\mathrm{CsI}(\mathrm{Tl})$ detectors. This has been verified involving other intrinsic features, like the resulting shapes of the energy spectra of LCPs measured by different detectors but at the same polar angle, or the spectra of the $\gamma$-rays.

In principle, the simulation of the PIM would require to carry out measurements of the scintillation light pulse-shapes for the CSI(Tl) crystals used, like it has been done in ref. [6], or with thin slices of the same $\mathrm{CsI}(\mathrm{Tl})$ scintillator material like in ref. [24]. On the other hand, 
as has been demonstrated above, the measured PIMs could be reproduced by simulations even when based on relatively rough approximations for $R(E, q)$ and $\tau_{f a s t}(E, q)$, for example with the expressions

$$
\begin{gathered}
R(E, q)=0.2227(E / q)^{1 / 3} \\
\tau_{\text {fast }}(E, q)=390+200(E / q)^{1 / 3},
\end{gathered}
$$

and the precision of the energy scales obtained for the LCP spectra is comparable with the energy resolution of the $\mathrm{CsI}(\mathrm{Tl})$ detectors.

The uncertainty of $E_{d}$ is naturally largest for lowest particle energies. On the other hand, at the FOBOS detector, the energy losses of the LCPS in penetrated layers of other detector materials are in this case larger than the residual energies $E_{d}$. Thus the necessary corrections for the energy losses introduce the dominating part of uncertainty into $E_{t}\left(L_{f a s t}\right)$ at small energies, and the error of $E_{d}$ can be neglected.

The limitation of the shaping time to $3 \mu \mathrm{s}$ in the measurement of the total light output of $\mathrm{CsI}(\mathrm{Tl})$ in ref. [15] does not significantly affect the final result for energies larger than a few $\mathrm{AMeV}$. At lower energies the slight distortions have been taken into account.

The influence of the rise-time of the scintillation light pulse can be neglected $\left(\tau_{\text {front }}=0\right.$, $h_{\text {front }}=0$ ), if the time gate $\Delta t_{\text {fast }}$ does not cover the initial part of the CsI(Tl) detector signal. As reported in ref. [6], the rise-time for electrons is about $40 \mathrm{~ns}$, and even shorter for high-ionizing particles, but the timing properties of the photomultiplier used have to be taken into account.

As already mentioned, the actual properties of the CSI(Tl) crystals used may be slightly different from those crystals investigated in ref. [6], and the parameters in eqs. $(2 \div 4)$ may not ideally suit to our simulations. A general adjustment of these parameters was supposed to be performed by fitting the simulated PIM to the measured one. It, however, turned out that this was not necessary on the level of accuracy required. Agreement of simulated and measured PIMs was achieved with $\tau_{\text {slow }}=4 \mu \mathrm{s}$. Such a value for the slow decay constant has likewise been found for CsI(Tl) crystals delivered by the same manufacturer [26].

The calibration procedure developed has a number of advantages:

(i) Special calibration measurements are not necessary.

(ii) It does not rely on measurement of the total light output.

(iii) All $210 \mathrm{CsI}(\mathrm{Tl})$ detectors can be calibrated in an unique manner by scaling the individual PIMs to an "ideal" PIM.

(iv) It enables a high degree of automation for data processing.

(v) Some visual inspection and check of data quality can be easily performed during the data processing.

This method has been applied for the calibration of the CsI(Tl) detectors of the scintillator shell of the FOBOS array in experiments carried out to investigate the decay of hot heavy nuclei produced in the reactions ${ }^{14} N(53 \mathrm{AMeV})+{ }^{232} \mathrm{Th}$ and ${ }^{197} \mathrm{Au}[27]$. Calibrated $\alpha$-particle spectra measured in these experiments [28] are shown for illustration in fig. 10.

\section{Acknowlegements}

The calculations of the energy losses of the LCP in the inner detector shells of FOBOS have kindly been performed by C.-M. Herbach.

The FOBOS project has been supported by the BMBF, Germany, under the contract Nr.: 06 DR 671. 


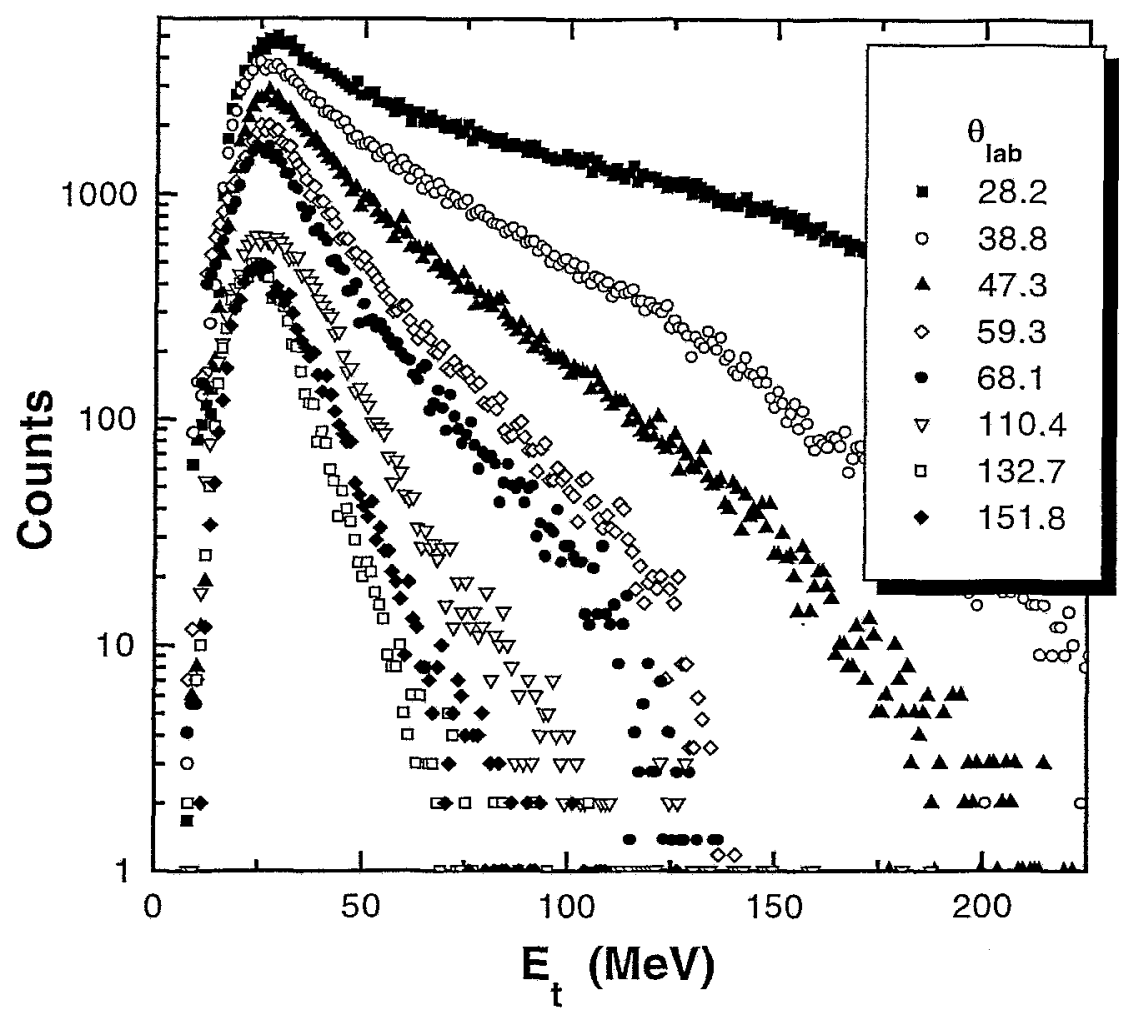

Figure 10: $\alpha$-particle spectra from the reaction ${ }^{14} N(53 \mathrm{AMeV})+{ }^{232} \mathrm{Th}$ accumulated into $\Delta \Omega=0.16$ sr for each laboratory angle $\vartheta_{l a b}$ ( $40 \%$ of the entire data body).

\section{References}

[1] J.B. Birks, "The Theory and Practice of Scintillation Counting", Pergamon Press, Oxford, 1964 .

[2] V.V. Avdeichikov et al., Nucl. Instr. and Meth. A349 (1994) 216

[3] S. Usuda, A. Mihara and H. Abe, Nucl. Instr. and Meth. A321 (1992) 247

[4] W. Wagner et al., Scientific Report 1991/92 "Heavy Ion Physics" (ed. B.I. Pustylnik) JINR E7-93-57, Dubna, Russia, 1993, p. 244.

[5] Proc. of the FOBOS workshop '94, Cracow, Poland, 1994 (ed. W. Wagner) Report FZR65, Rossendorf, Germany, 1995;

M. Andrassy et al. and FOBOS Collaboration, JINR E7-95-148, Dubna, Russia, 1995; W. Wagner et al. and FOBOS Collaboration, JINR E13-97-118, Dubna, Russia, 1997; H.-G.Ortlepp et al. and FOBOS Collaboration, FZR-181, Rossendorf, Germany, 1997. (submitted to Nucl. Instr. and Meth.)

[6] F. Benrachi et al, Nucl. Instr. and Meth. A281 (1989) 137 
[7] Y. Larochelle et al., Nucl. Instr. and Meth. A348 (1994) 167

[8] K. Michaelian, A. Menchaca-Rocha, and E. Belmont-Moreno, Nucl. Instr. and Meth. A334 (1993) 457

[9] H. Fuchs, D. Kamanin, and J. Krüger, Ann. Rep. 1994, HMI-B 526, Hahn-Meitner-Institut, Berlin, 1995, p. 137.

[10] A.S. Fomichev et al., Nucl. Instr. and Meth. A344 (1994) 378

[11] A.J. Bird et al., Nucl. Instr. and Meth. A353 (1994) 46

[12] M. Moszynski et al., Nucl. Instr. and Meth. A336 (1993) 587

[13] J. Alarja et.al., Nucl. Instr. and Meth. A242 (1986) 352

[14] A.S. Fomichev et al., Nucl. Instr. and Meth. A350 (1994) 605

[15] D. Horn et al., Nucl. Instr. and Meth. A320 (1992) 273

[16] N. Colonna et al., Nucl. Instr. and Meth. A321 (1992) 529

[17] K. Michaelian, A. Menchaca-Rocha, and E. Belmont-Moreno, Nucl. Instr. and Meth. A356 (1995) 297

[18] W. Wagner and D.V. Kamanin, Report FZR-130, Rossendorf, Germany, 1996, p. 150.

[19] W. Wagner et al., Scientific Report 1993/94 "Heavy Ion Physics" (ed. B.I. Pustylnik) JINR E7-95-227, Dubna, Russia, p. 221.

[20] A.A. Aleksandrov et al., Instruments and Experimental Techniques, vol. 40, No. 2, 1997, p. 170 .

[21] W. Wagner et al., JINR Rapid Comm. 4(61)-93, Dubna, Russia, 1993.

[22] W. Wagner et al., Report FZR-78, Rossendorf, Germany, 1995, p. 117.

[23] J. Henniger and B. Horlbeck, JINR E6-84-366, Dubna, Russia, 1984.

[24] T. Masuda et al., Nucl. Instr. and Meth. A332 (1992) 135

[25] W. Wagner and H.-G. Ortlepp, Report FZR-35, Rossendorf, Germany, p. 93.

[26] K.R. Schubert, Technische Universität Dresden, Germany (private communication)

[27] H.-G. Ortlepp et al. and FOBOS Collaboration, Scientific Report 1995/96 "Heavy Ion Physics", (ed. B.I. Pustylnik) JINR E7-97-206 JINR Dubna, Russia, p. 236.

[28] D.V. Kamanin et al., Proceedings of the Third Int. Conf. on Dynamical Aspects of Nuclear Fission, Casta-Papiernicka, Slovak Republic 1996, (ed. by J. Kliman and B.I. Pustylnik) JINR E6,7-97-49, Dubna, Russia, p. 183. 